\title{
Gamma-rays from Blazars and the Extragalactic Background Light (EBL)
}

\author{
Luigi Costamante* \\ Author affiliation: INAF-OAB, via E. Bianchi 46, 23807 Merate, LC \\ E-mail: luigic2011@gmail.com
}

Gamma-rays from blazars at high energy (HE, 0.1-100 GeV) and very high energy (VHE, >0.1 $\mathrm{TeV}$ ) have constrained the intensity and spectrum of the diffuse Extragalactic Background Light (EBL) to be close to the level given by galaxy counts, and in agreement with the most recent models of galaxy evolution. As a consequence, they indicate the starlight from normal galaxies as the predominant origin of the EBL, excluding a strong contribution from other sources like PopIII stars. A low EBL means that future detections at VHE of sources as far as $z=1$ or even 1.5 are indeed possible and expected, without invoking new and exotic physics. The combination of HE and VHE spectra has also allowed to put important lower limits on the intergalactic magnetic field (IGMF), from the absence of evidence for the secondary emission expected from the electronpositron pairs produced in gamma-gamma collisions with the EBL photons. However some issues remain open, in particular in the mid and far-infrared bands and in the blazar spectra at multi-TeV energies. This energy range, probed by the new and upcoming facilities like ASTRI, HAWC and CTA, remains the most promising for significant improvements in our understanding of both the EBL and blazar physics.

XI Multifrequency Behaviour of High Energy Cosmic Sources Workshop 25-30 May 2015

Palermo, Italy

\footnotetext{
* Speaker.
} 


\section{Introduction}

Gamma-rays interact with photons of lower energies by photon-photon collision, if above threshold for pair production. The primary radiation beam is then depleted by a net absorption effect, while the converted energy is reprocessed by the created pairs and dispersed in the ambient medium. Since the discovery of the Cosmic Microwave Background (CMB), it has been realized that the optical depth can become very high on extragalactic distances, even for the more tenuous diffuse background in the $0.1-100 \mu \mathrm{m}$ range produced by all stars and dust in the Universe (i.e. the EBL). The gamma-ray spectrum of extragalactic sources can be dramatically affected by this absorption, but this provides also an effective way to probe the intensity and spectrum of these diffuse backgrounds $[1,2,3,4]$.

The EBL is formed by all the radiative activity of the Universe throughout cosmic history, and thus carries unique information about the formation and evolution of galaxies. Its spectral energy distribution (SED) is expected to be dominated by two main humps [5], produced by the direct starlight from galaxies at Optical to near-infrared (NIR) wavelengths, and by the light reprocessed by dust at mid- to far-infrared (MIR-FIR) wavelengths (Fig. 1). The direct measure of the EBL from the night-sky background is highly uncertain due to strong foregrounds [5], mainly zodiacal light [6]. Robust lower limits on its intensity can instead be derived from integrating galaxy counts down to the faintest end of the luminosity functions [7, 8, 9, 10, 11, 12].

Blazars are the most luminous persistent sources of gamma-rays in the Universe and can have spectra extending up to tens of $\mathrm{TeV}$. Thus they provide an independent way to probe the EBL by studying the imprint of the EBL attenuation on their gamma-ray spectrum, which depends on EBL intensity and spectral shape. However, blazars are not standard candles, being highly variable both in time and spectral properties $[13,14,15]$, and with jet composition and emission mechanisms not yet fully known $[16,17]$.

The study of the EBL through gamma-rays constitutes a classic problem of one equation with two variables [18] (even three when redshift is unknown). Some assumptions therefore need to be introduced, or a check of the degeneracies in combined fits. So far, the methods used to derive upper limits or tentative measures of the EBL have been based on one of the following three approaches: 1) to identify a realistic range of blazar SED properties, and exclude EBL shapes which give odd values (mainly hardness limits or sudden up-turns or pile-ups); 2) to extrapolate the spectrum from lower energies (from the Fermi-LAT band alone or by a physical model of the whole SED); 3) to fit both gamma-ray spectra and EBL together, with some boundary conditions, hoping that the maximum likelihood would correspond to the true value of both (for a full review, see e.g. [19]).

\section{EBL imprint onto gamma-ray spectra}

The convolution of the $\gamma-\gamma$ cross section with the EBL spectrum yields an energy-dependent attenuation. The two-humped nature of the EBL SED gives a complex but unique and specific shape (Fig. 1), especially clear in single spectra covering the widest energy band (e.g. from 0.1 to $10 \mathrm{TeV})$. A few important points can be noted:

1) the optical depth $\tau$ mostly increases with gamma-ray energy, steepening the observed gamma-ray spectrum with respect to the intrinsic one (namely, the photon index $\Gamma_{o b s} \geq \Gamma_{i n t}$ ). How- 

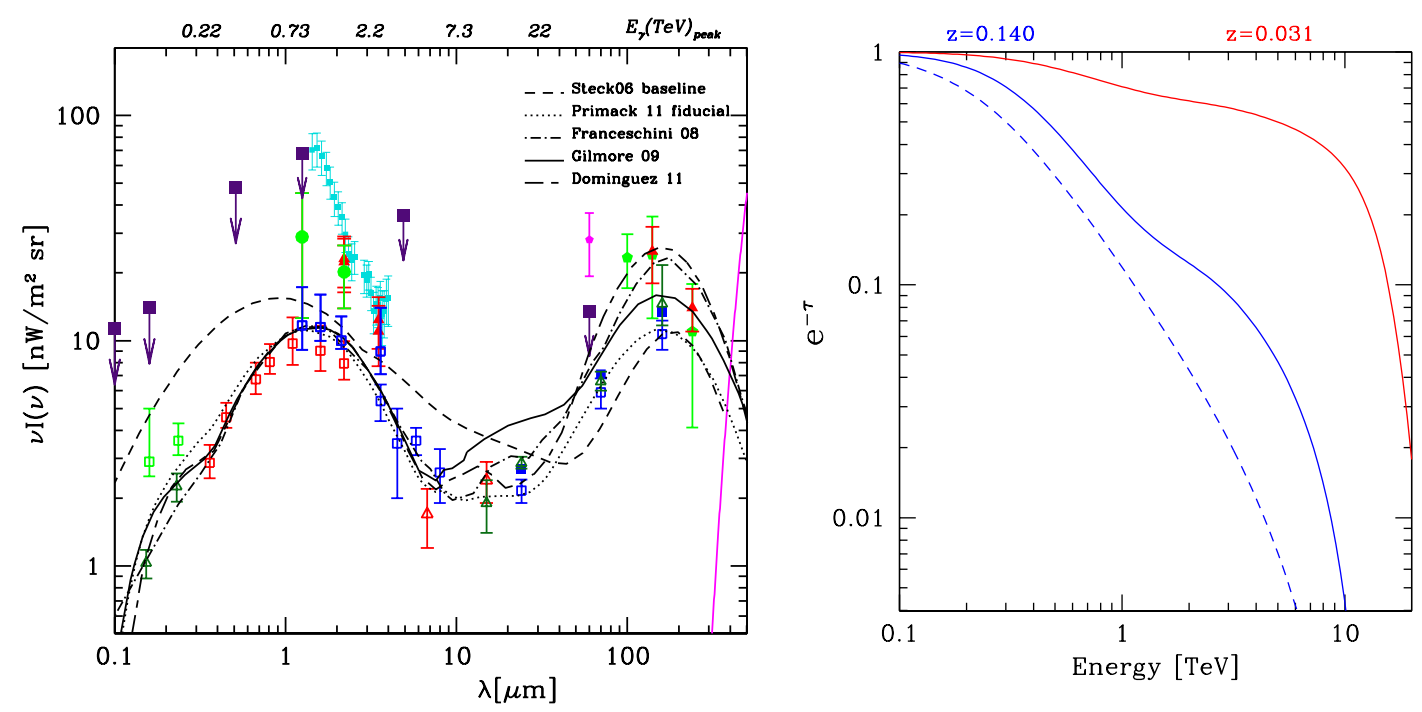

Figure 1: Left: SED of the EBL. EBL data are from review compilations[5, 19]. Open symbols correspond to lower limits from galaxy counts, while filled symbols correspond to direct estimates. The curves show a sample of different recent EBL models, as labelled [20, 21, 22, 23, 24]. On the upper axis it is plotted the $\mathrm{TeV}$ energy corresponding to the peak of the $\gamma-\gamma$ cross section. Right: attenuation curves $e^{-\tau}$ for sources at two different redshifts (solid lines) with the same EBL model [23], and at the same redshift ( $\mathrm{z}=0.140$ ) but for two different EBL models (dashed line for Stecker06 [24], solid lines for Dominguez11 [23]). It is assumed $H_{0}=70 \mathrm{~km} / \mathrm{s} / \mathrm{Mpc}, \Omega_{m}=0.3, \Omega_{\Lambda}=0.7$.

ever, the steepening is different in different gamma-ray bands, due to the change of EBL slope (peaks or valley of the SED). This can be seen in Fig. 1, where the attenuation factor $e^{-\tau}$ is plotted as a function of energy. Over some energy ranges (namely $0.2-1 \mathrm{TeV}$ and 2-8 TeV), it approximates a power-law shape. Thus, if the intrinsic spectrum is a power-law, the observed spectrum in that range can also be well fitted by a power-law, with steeper index.

2) The amount of steepening $\Delta \Gamma$ increases both with distance and EBL intensity. Thus redshift gives leverage: the same uncertainty in EBL flux $\Delta F_{\mathrm{EBL}}$ causes a higher $\Delta \Gamma$ at larger redshifts. More distant sources provide therefore more sensitivity for EBL constraints, but at the same time they suffer stronger attenuation, yielding much lower statistics. As a result, there is an optimal range of redshifts which provides the best compromise, depending on the EBL waveband to be probed: sources at $z<0.05$ are best for the highest $\mathrm{TeV}$ energies (probing longer EBL wavelengths, dominated by dust emission), while sources at $z \sim 0.15-0.25$ provide the best compromise for the most stringent EBL limits in the 0.3-8 $\mathrm{TeV}$ range.

3) If the EBL number density $n(\varepsilon) \propto \varepsilon^{-\beta}$ (i.e. the EBL SED $\propto \lambda^{\beta-2}$ ), the optical depth $\tau\left(E_{\gamma}\right)$ becomes $\propto E_{\gamma}^{\beta-1}$. Where $\beta \approx 1$, the optical depth becomes independent of energy [18]. In such case there is no steepening: the absorbed spectrum reproduces the original shape, simply attenuated by a constant factor. The EBL is close to that value between 3 and $10 \mu \mathrm{m}$, according to all most recent EBL models [20, 23, 21, 42]. This causes a unique flattening feature in the attenuation curve between $\sim 1-2$ and $8-9 \mathrm{TeV}$, where the observed gamma-ray spectrum partly recovers its intrinsic slope and thus can appear harder than in the $0.2-1 \mathrm{TeV}$ band even when the emitted spectrum is not. 

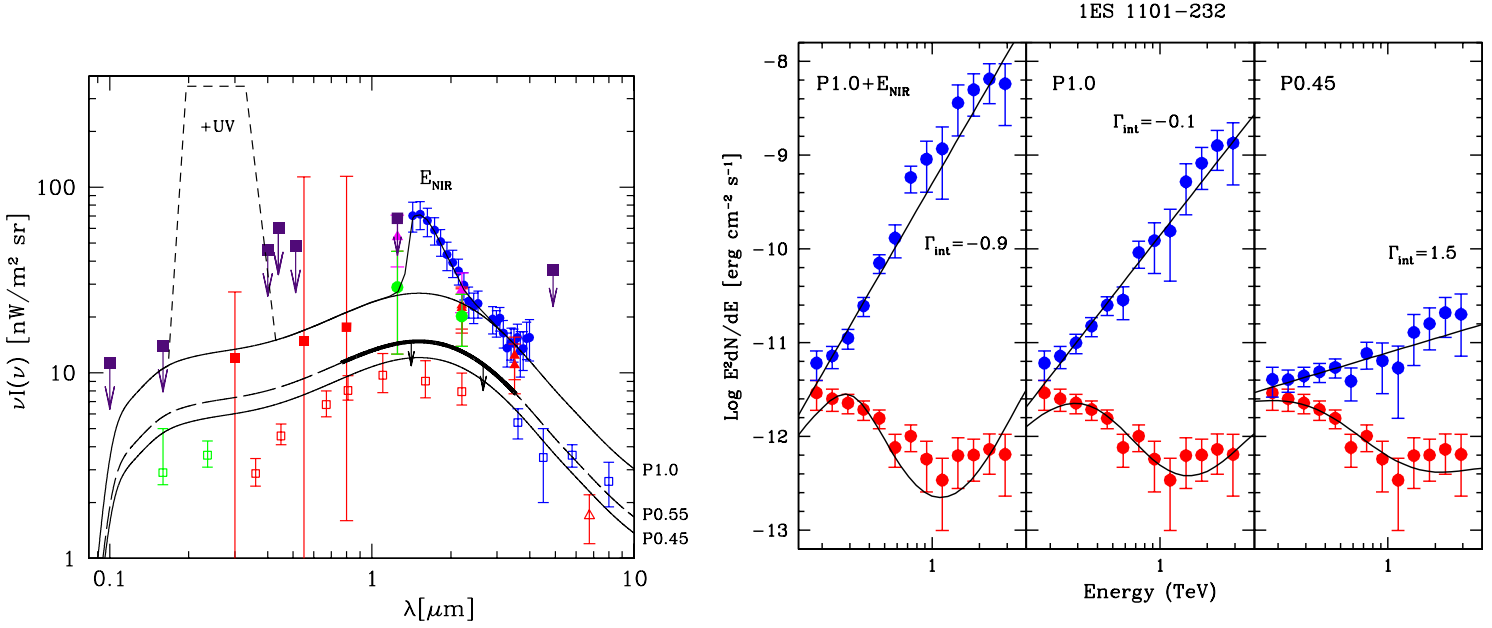

Figure 2: Left: SED of the EBL with test EBL shapes and upper limits curves derived from the assumption $\Gamma_{\text {int }} \geq 1.5$ (black marked region). Right: the HESS spectrum of 1ES 1101-232, corrected for absorption with three different EBL shapes, as labeled. It shows how reducing the EBL intensity softens the required intrinsic spectrum. Lower (red) points: observed data. Upper (blue) points: absorption-corrected data. The lines show the best fit power-laws to the reconstructed spectrum, and corresponding shape after absorption (Figures from [25]).

4) Therefore, NO cutoff is produced by EBL absorption between 0.2 and $8 \mathrm{TeV}$ ! Any cutoff or steepening seen in this energy range is intrinsic to the source spectrum. The two cutoffs expected by EBL absorption occur only between the unabsorbed and absorbed parts of the gamma-ray spectrum for cosmological distances (i.e. between 50 and $500 \mathrm{GeV}$ ), and above $8-10 \mathrm{TeV}$. That is, at the onset of the starlight and dust EBL peaks, respectively. The first cutoff is best sampled by low gamma-ray energy instrument (Fermi-LAT and low-threshold Cherenkov arrays) with high-redshift sources, while the highest cutoff by $\mathrm{TeV}$ instruments with the highest effective area above few $\mathrm{TeV}$ and low-redshift sources.

\section{Constraints on the starlight EBL}

Until 2005, the data were quite ambiguous: an observed VHE spectrum could be the result of either a hard intrinsic spectrum attenuated by a high EBL, or a soft intrinsic spectrum less absorbed by a low EBL. The required intrinsic VHE spectrum was always within the typical range of spectra shown by blazars. This ambiguity undermined also our capability to study blazars' emission, since it implied large differences both in energy and luminosity of the gamma-ray peak in their SED.

\subsection{Optical-IR constraints}

The breakthrough occurred in 2005 with the HESS observations of the BL Lac objects 1ES 1101$232(z=0.186)$ and H 2356-309 ( $z=0.165$ ) [25]. The observed gamma-ray spectra (detected between 0.2 and 1-3 TeV) were much harder than expected given their redshift, implying extremely hard intrinsic spectra $\left(\Gamma_{i n t} \lesssim 0\right)$ in case of high EBL densities (Fig. 2). Such hard spectra were never seen in the closer, less absorbed objects and were at odds with all the known blazar physics and phenomenology. They were also not supported by same-epoch multiwavelength observations of 

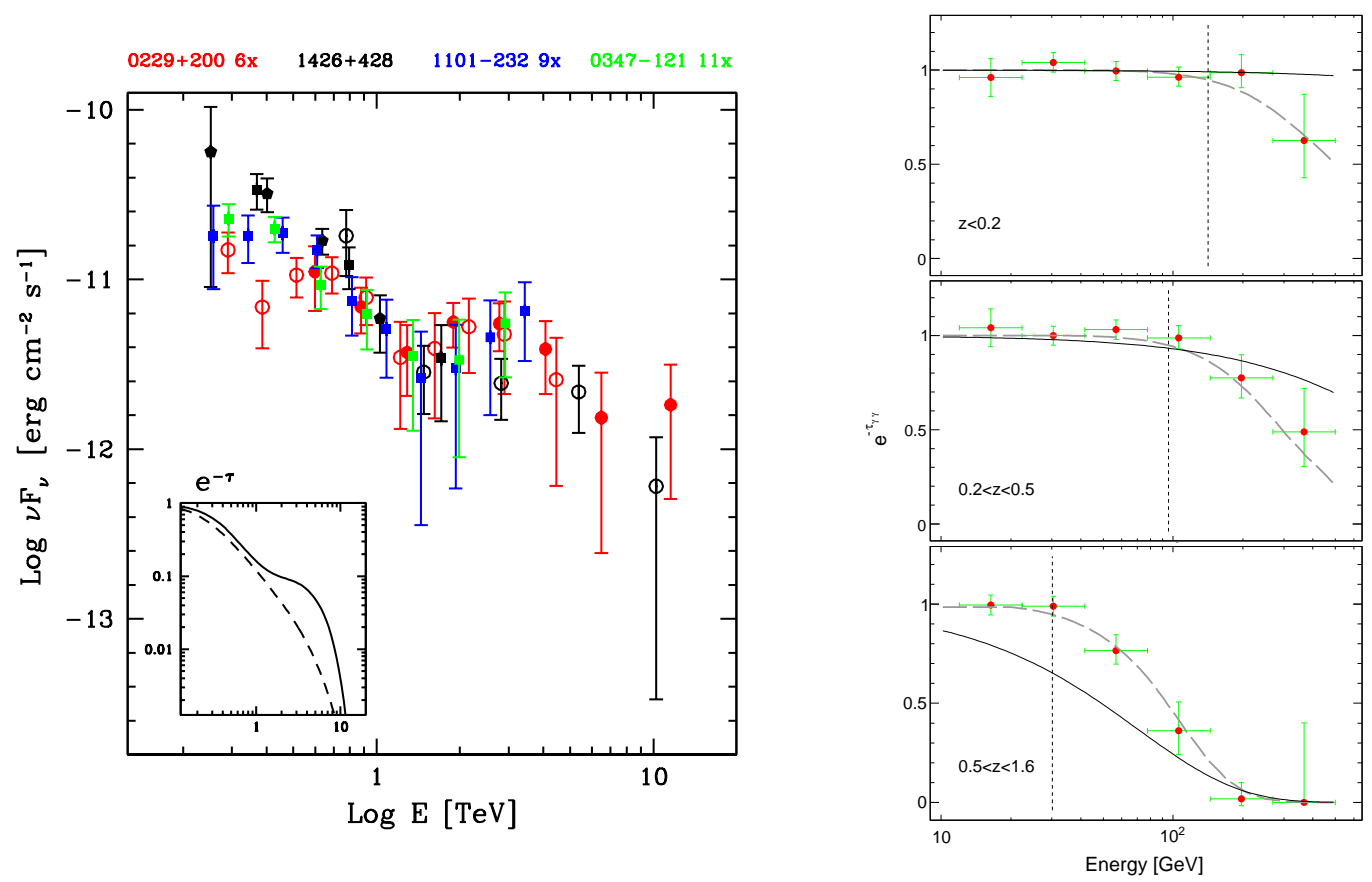

Figure 3: Left: Observed VHE spectra of objects detected above 1-2 TeV, rescaled as labeled to match the flux of 1ES 1426+428 around 0.8-1 TeV. Above $1 \mathrm{TeV}$ the data lie above the extrapolation from lower energies, showing the flattening feature expected from absorption with an EBL from standard galaxy evolution models, instead of the increased steepening expected from a flatter EBL spectrum [24]. From [19]. Right: ratio of the average extrapolated vs observed Fermi-LAT spectra in different redshift bins. It shows a cut-off as a function of increasing redshift. The vertical line marks the critical energy $E_{c r i t}$ (see text). Dashed curves show the attenuation expected from the EBL absorption (with model by [20]), The thin solid curve shows the intrinsic cut-off scenario, i.e. the best-fit model assuming that all the sources have an intrinsic exponential cut-off and follow the "blazar sequence" model [32, 15]. From [31].

their SED, and a high EBL would have implied a dramatic change of properties within a very narrow range of redshifts. A low EBL intensity could instead accomodate all the new results within the typical range of blazar properties. Assuming that the intrinsic spectra were not harder than $\Gamma=1.5$, an upper limit could be derived, which resulted very close to the lower limits given by the integrated light of galaxies [25]. This result has been later confirmed by several other sources and observations (e.g. [26, 27, 28]).

Furthermore, the HESS detection of 1ES 0229+200 [29] $(z=0.140)$, with good statistics up to $\sim 10-15 \mathrm{TeV}$, extended this result in the MIR range of the EBL, confirming the previous finding from the HEGRA spectrum of 1ES 1426+428 [30]. Taking advantage of the constraints at 1-2 $\mu \mathrm{m}$, the 1ES $0229+200$ spectrum limits the EBL SED between 2 and $10 \mu \mathrm{m}$ to be $\propto \lambda^{-\alpha}$ with $\alpha \gtrsim 1.1 \pm 0.25$ [29], yielding a flux upper limit again very close to the lower limits given by galaxy counts from Spitzer [11, 12]. In fact, combining the data of all experiments, the overall 0.2-10 TeV spectrum of these two objects showed for the first time the typical flattening feature expected from a starlight-dominated EBL spectrum (Fig. 3, left).

\subsection{UV-Optical constraints}

The second strong evidence for a low EBL has come from the Fermi-LAT spectra at high energies 
which probe the UV-optical part of the EBL and the onset of EBL absorption above $30-50 \mathrm{GeV}$. In the local universe $(z<0.2)$, the Fermi-LAT band is essentially insensible to EBL attenuation, but the absorption edge shifts more and more into the LAT band as redshift increases $\left(E_{\text {crit }}(z) \approx\right.$ $170(1+z)^{-2.38} \mathrm{GeV}$, where $E_{\text {crit }}$ is the critical energy below which less than $5 \%$ of the source photons are absorbed [31]). It was then possible to try to detect the first EBL absorption edge in the combined gamma-ray spectra of all blazars, as a cut-off that changes amplitude and energy with redshift. The Fermi-LAT Collaboration [31] analyzed 46 months of LAT data in the 1-500 GeV band, for a subset of 150 blazars with a significant detection above $3 \mathrm{GeV}$ and known redshift. The sample covers a redshift range $0.03-1.6$, meaning $E_{\text {crit }}$ is always $>25 \mathrm{GeV}$. The intrinsic spectrum of the source was determined by fitting the unabsorbed part $\left(1 \mathrm{GeV}-E_{c r i t}\right)$, and then extrapolating to higher energies. By combining all the spectra in a maximum likelihood fit, it was possible to determine the average deviation, above the critical energy, of the observed spectra from the extrapolated ones.

The result is the detection of an average cut-off feature in the blazar spectra [31], whose amplitude and modulation in energy evolve with redshift as expected for absorption on a lowdensity EBL (Fig. 3, right). This feature is consistent with low EBL models at a significance $>5 \sigma$ against the null hypothesis (i.e. no absoprtion at all), and excludes models with a larger EBL intensity as well, particularly in the UV [31] (above 5 $\sigma$ ).

In principle, this spectral cut-off could also be due to intrinsic properties of the gamma-ray sources, in total or in part. The sample composition (high vs low-energy-peaked BLLacs) indeed changes with redshift, and evolution effects or the end of the emitting particle distribution could play a role. However, if intrinsic, the cut-off is expected to evolve differently with redshift than is observed, assuming that blazars are distributed according to the blazar-sequence scenario [31]. Nevertheless, the close agreement between the intensity of the UV-Opt background as measured from the cut-off in Fermi and that due to galaxies individually resolved by the Hubble Space Telescope $[7,9]$ leaves little room both to an intrinsic origin of the cut-off feature and to any additional diffuse UV emission.

\section{Again an IR background - TeV gamma-ray crisis ?}

The EBL above $\sim 20 \mu \mathrm{m}$, towards the far-infrared hump of the EBL SED, is probed by gammarays above $10 \mathrm{TeV}$. So far the best spectra at these energies are still provided by the HEGRA data on Mkn 421 [33] ( $z=0.031)$ and Mkn 501 [34] $(z=0.034)$. The latter, in particular, was found in conflict with an initial high estimate of the EBL at $60 \mu \mathrm{m}$ [35], which would require a sharp pile-up feature at the end of the intrinsic $\mathrm{TeV}$ spectrum (Fig. 4, left). This problem was known as "IR background - TeV gamma-ray crisis" [36], and was resolved as an overestimate of the EBL flux. With the new strong limits at $10 \mu \mathrm{m}$, however, how the EBL rises towards the dust peak is critical, and there is less room for a gamma-ray compatible EBL. The Mkn 501 data show problems with the best and most recent EBL calculations, which cause again an up-turn or pile-up feature at the highest energies (see Fig. 4). The information on the warm-dust emission for the models and the statistic of the gamma-ray data are not yet sufficient to draw any sensible conclusion. More observations above 5-10 $\mathrm{TeV}$ are needed, achievable with the present or next generation of $\mathrm{TeV}$ telescopes (e.g. ASTRI [37] and CTA [38]). 

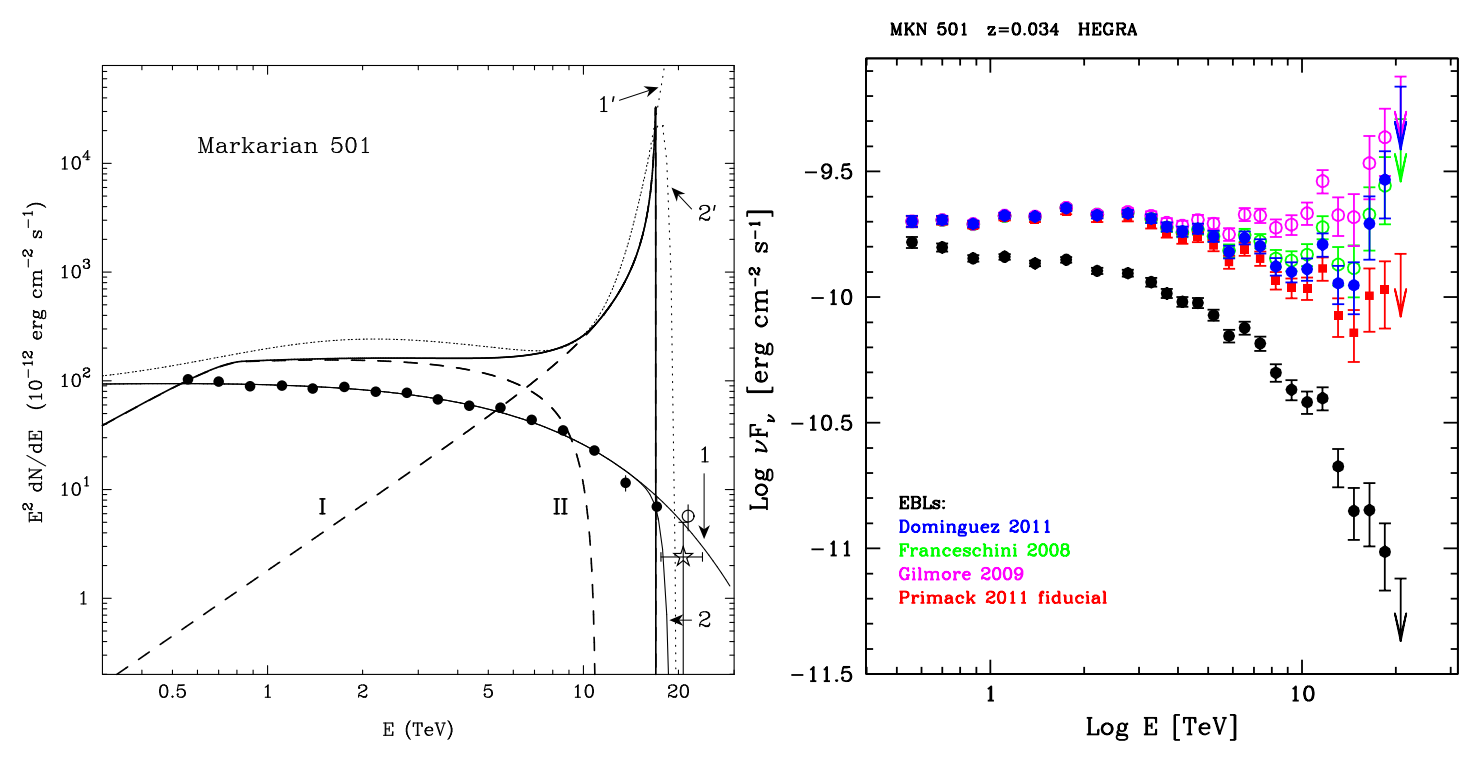

Figure 4: "Ancient" and "modern" versions of the "IR background - TeV gamma-ray" crisis[36]. Left: models of pile-up spectra to explain the HEGRA data of Mkn 501, if the highest $60 \mu \mathrm{m}$ EBL flux [35] were right. From [18]. Right: HEGRA data as observed [34] (black points) and corrected for absorption with 4 recent EBL models, as labeled [20, 21, 23, 22]. Except for the "Primack 2011 fiducial" model [22], all the others seem to cause an up-turn in the HEGRA spectrum.

\section{Combined Maximum Likelihood fits}

With more and more data, it becomes feasable to try to measure the EBL by fitting models of both the EBL attenuation and the intrinsic blazar spectrum together, by means of a maximum likelihood fit to the observed VHE spectra. The underlying assumption is that the true intrinsic spectrum plus true attenuation curve combined should maximize the likelihood. This study has been performed first on the HESS data [39], and then recently expanded and applied to a larger dataset [40]. The result of the fits confirms the presence of EBL absorption (at $\sim 8-11 \sigma$ above the null hypothesis, depending on the dataset), and gives a low-density EBL as best fit value, though slightly higher than the most recent EBL models [20, 23, 41].

However, this approach is undermined by two main problems: 1) given the complex interaction between the intrinsic spectrum and the shape of EBL attenuation, there is no guarantee that the combination of the two which maximizes the likelihood is the correct one. Indeed, among different objects the maximum probabilities occur at very different EBL normalizations, implying de facto that the true value is certainly not given by the peak of the $\chi^{2}$ probability (see Fig.1 in [39]). The systematic uncertainties therefore appear underestimated. And 2), the combination of the teststatistic (TS) functions to find the best-fit value makes the result, by construction, heavily biased towards datasets with higher statistics. In this case it is the datasets of PKS 2155-304 [39], which is characterized by a rather steep and intrinsically curved spectrum. This allows for a higher-than-real EBL density to be considered viable with a high TS, yielding a best fit which is biased towards a higher EBL.

Nevertheless, despite the dubious numbers, this result does indicate that on average the blazar VHE spectra are better fitted including some amount of EBL absorption rather than not, thus sup- 
porting the Fermi-LAT result against the null hypothesis. This is precisely the expected effect of the flattening feature shown in Fig. 3.

Another approach is to predict the unattenuated intrinsic spectrum of blazars in the VHE band by fitting a physical model to the blazars jet SED from infrared to $\mathrm{GeV}$ gamma-rays, taking advantage of the improvements in the sampling of the SED from simultaneous multiwavelength observations. The most complete study of this type has been performed by Dominguez et al. [43], who applied a homogeneous synchrotron Self-Compton (SSC) model to 15 blazars and derived the EBL optical depth from the ratios between the predicted and detected VHE fluxes. The results confirm the presence of a low EBL and are in agreement with the most recent EBL models [20, 23, 41]. The main problem with this approach is that a single-zone SSC model is often not adequate and can fail to reproduce the blazars SED properties, especially the spectra in the gamma-ray hump of some $\mathrm{TeV}$ blazars. Observations show that, for a given $\mathrm{GeV}$ spectrum and overall $\mathrm{SED}$, the range of possible VHE spectra is still large $(\Delta \Gamma \approx 2)$, even theoretically in a SSC scenario. One-zone models can work a-posteriori, but do not work reliably a-priori. This problem can be mitigated as the number of sources increases. On average, however, where the SSC model does seem to work, the results confirm a low level of the EBL.

\section{Consequences of a low EBL}

Despite some shortcomings, the different methods and analyses using both $\mathrm{GeV}$ and $\mathrm{TeV}$ gamma-rays all converge towards one coherent picture, which prescribes a low intensity of the EBL (at least in the waveband 0.1-10 $\mu \mathrm{m}$ ), as described by the most recent EBL models from galaxy evolution [20, 23, 41].

This convergence has three main consequences: 1) it pins down the origin of the EBL, showing that at these wavelengths it is strongly dominated by the direct starlight from galaxies, and excludes a strong contribution from other sources like Pop-III stars (e.g. [44]). Indeed the Fermi-LAT data can put an upper limit on the co-moving star formation rate of Pop-III stars at $\leq 0.5 \mathrm{M}_{\odot} \mathrm{Mpc}^{-3}$ $\mathrm{yr}^{-1}$, and a redshift $z \geq 10$.

2) It means that the intergalactic space is more transparent to gamma-rays than previously thought, thus enlarging the gamma-ray horizon. Detections of objects at $100-300 \mathrm{GeV}$ are well possible even from $z \sim 1$, also up to $\sim 500 \mathrm{GeV}$ during flares, without resorting to any kind of new or exotic physics. In fact, considering the detection at VHE of FSRQ objects and the fluxes with hard spectrum showed by 3C 454.3 during some flares [45], even 3C 454.3 itself ( $z=0.859)$ could be detectable at VHE by present Cherenkov telescopes, and objects with luminosities $10^{48-49} \mathrm{erg} \mathrm{s}^{-1}$ up to $z \sim 1.5$ in the $80-200 \mathrm{GeV}$ band.

3) It strongly reduces the ambiguity on blazars $\mathrm{TeV}$ spectra, allowing the full study of their gamma-ray properties as a population, without biases.

\section{Secondary emission}

The energy deposited by the primary $\gamma-\gamma$ interaction is not lost, but is reprocessed at lower energies by inverse Compton of the produced pairs with CMB photons, again at gamma-ray energies, with typical values $E_{\gamma} \sim 1\left(E_{0} / 40 \mathrm{TeV}\right)^{2} \mathrm{TeV}$ [46]. 

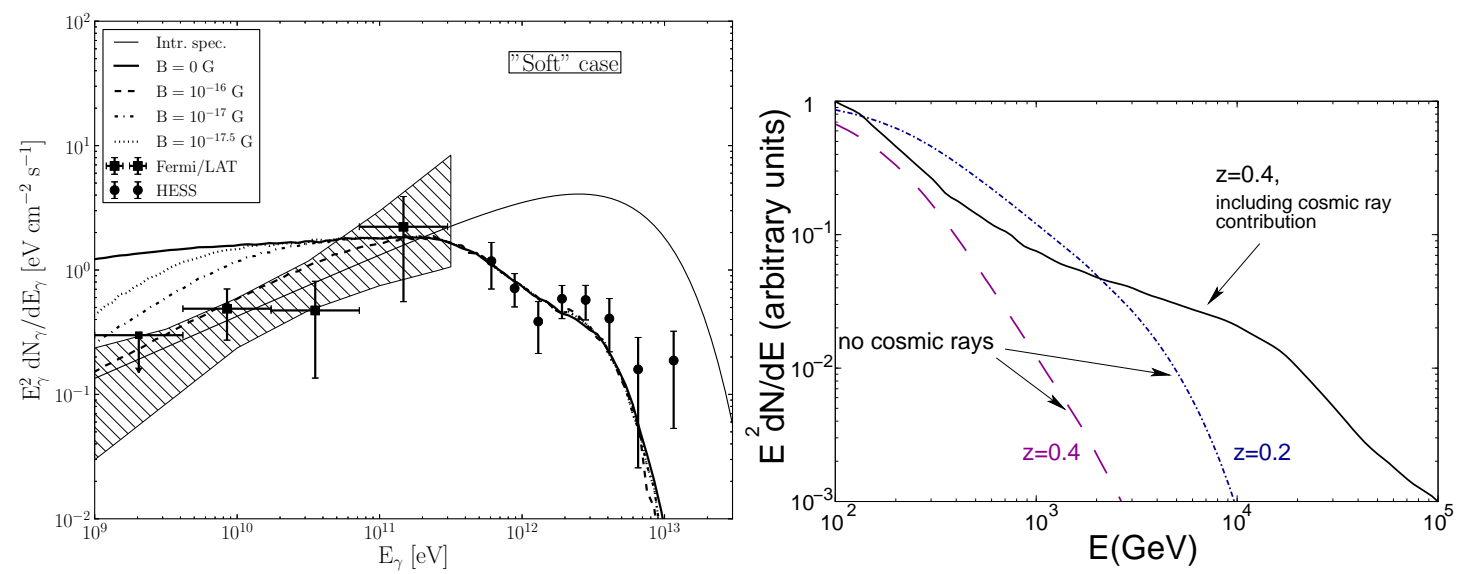

Figure 5: Left: constraints to the integralactic magnetic field by the presence or absence of the reprocessed TeV flux re-emerging in the Fermi-LAT band. From [53]. Right: spectrum of primary+secondary gamma rays produced in line-of-sight interactions of UHE protons vs only primary emission. Since most of the observed photons are produced closer to the observer, there is less attenuation due to EBL absorption and a significant flux at multi-TeV energies can still be present. From [59].

For photons above $\sim 20-50 \mathrm{TeV}$, the absorption length is typically short, the pairs are produced within few $\mathrm{Mpc}$ of the source, in regions where the magnetic field is still relatively high, and thus can develop an electromagnetic cascade which surrounds the source with a giant, isotropically emitting halo of pairs [46]. No firm evidence for such pair halos has been obtained so far, though hopefully their emission could be within reach of the future Cherenkov telescope CTA [38].

Below $\sim 10-20 \mathrm{TeV}$, instead, the pairs are produced predominantly in the intergalactc space and the secondary emission occurs in the GeV domain [47], where the Universe is transparent. Thus only one conversion $\gamma$-pairs $-\gamma$ occurs. The intensity of the IGMF determines if the secondary gamma-rays are produced within the emission cone of the primaries (enhancing the observed emission) or are dispersed in the background. This provides a very effective way to measure the IGMF: the comparison of the VHE spectra (which determines the absorbed energy flux depending on EBL intensity) with the HE ones (where the absorbed energy flux should reappear) can put limits on the poorly known strength of the IGMF [47, 48, 49].

The Fermi-LAT spectra and upper limits obtained so far on several TeV BL Lacs (but in particular 1ES $0229+200$ ) allow a lower limit to the IGMF intensity to be put at a level of $B \gtrsim 10^{-17}$ Gauss $[48,50,51,52,53]$.

\section{Blazars as sources of UHE cosmic rays?}

Is there a way to circumvent the severe EBL absorption from cosmological distances? Without invoking new or exotic physics, a possibility is given if blazars are also sources of ultra-highenergy (UHE) protons. Protons below the GZK cutoff $\left(E \lesssim 5 \times 10^{19} \mathrm{eV}\right)$ do not lose a significant part of their energy in interactions with background photons, but deposit a small amount of their energy by pair-production collisions with CMB photons, initiating an electromagnetic cascade. Secondary gamma-rays are produced all along the path, but because they are generated closer to the observer, they are correspondingly less suppressed by EBL absorption. If the IGMF intensity 
is low enough along some line of sights, the broadening of both the proton beam and the cascade electrons can be less than the point spread function of the Air-Cherenkov detectors $\left(\sim 0.1^{\circ}\right)$. For distant sources $(z \gtrsim 0.5)$ and $\mathrm{TeV}$ energies, the secondary emission can become dominant over the primary emission, the latter being filtered out by the full EBL suppression (Fig. 5).

This mechanism has been first invoked to explain some TeV spectra of blazars [54, 55]. The present VHE data do not support this scenario (see e.g. [19]), and it seems unlikely for a random line of sight to Gpc distances not to cross any region of the Large Scale Structure with "high" (nano-Gauss) magnetic field. The filling factor of such fields might be significant given the typical size of the voids (30-100 Mpc [56]) and considering that the magnetic field seems to follow the mass density as $B \propto \rho^{2 / 3}$ [57]. However, if in the future objects at high redshift are detected at TeV energies, this scenario could provide the only viable astrophysical interpretation consistent with conventional physics [58], provided that the energetics, spectral and temporal behavior of the data are consistent [59]. Given the importance and far-reaching implications of a detection of gammarays above $1 \mathrm{TeV}$ from $z=1$ for example, dedicated observational campaigns on $\mathrm{TeV}$ candidates with established large redshift seem fully justified, as well as a sky survey with the largest possible sensitivity and collection area in the multi-TeV range, like the south array of CTA [38].

\section{Conclusions}

The data provided by Cherenkov telescopes and the Fermi satellite have brought substantial progress in the study of the EBL through gamma-rays. Together with the lower limits from the integrated light from galaxies, the VHE spectra of hard BL Lacs and the collective signal in the Fermi-LAT band have pinned down the EBL in its direct starlight component to a level better than $10-15 \%$, and to a low density as produced by standard galaxy evolution models. As a consequence, also the average intensity of the IGMF can be constrained to $B \gtrsim 10^{-17} \mathrm{G}$.

It is particularly important to stress that, at the time of writing, all gamma-ray data from blazars are fully consistent with this simple picture, and as pure primary emission. Other more complicated or exotic explanations, though within the realms of possibility, are neither required nor suggested by the data. All new detections are conforming so far with this low-density EBL scenario.

Given the small room left between upper and lower limits, more and better data are expected to provide confirmations but no further improvements in the EBL stellar hump. The limit is essentially represented by the systematic uncertainty given by our ignorance in the details of blazar physics and jet physical conditions. To this respect, the precision with which some simulations could in principle improve the EBL measurement might be deceptive, being far from a comparable accuracy.

The most sizeable progress and new results are likely to come from gamma-ray spectra in the $\sim 1-100 \mathrm{TeV}$ range, as allowed for example by the upcoming mid and small-telescopes array in CTA [38], its precursor ASTRI project [37], and the HAWC detector [60].

\section{Questions:}

Q. (P. Biermann): This conflict between EBL and TeV giving very low magnetic fields in the proton-cascade model is accentuated by the cosmic simulations that derive the magnetic field selfconsistently, as done by Ryu et al. (ApJ, Science). These simulations do not include the effect 
of energy from radiogalaxies. However, once you include radiogalaxies, the magnetic field gets compressed into thin sheets. Allowing for very thin sheets, the arguments by Essey \& Kusenko (2010+) and Ryu et al. (2008+) can be compatible. But this solution requires very thin sheets, not implausible in plasma physics.

A.: Yes, right. It could work if the filling factor is strongly reduced. However, the magnetic field in the compressed thin sheets cannot be too high, because then even one passage could be sufficent to deflect the primary proton beam away from the line of sight to the source.

\section{References}

[1] A.I. Nikishov, Soviet Phys. JETP 14393 (1962)

[2] J.V. Jelley, Physical Review Letters 16479 (1966).

[3] Gould R.J. \& Schréder G.P., Physical Review 155, 1408 (1967).

[4] F.W. Stecker et al., ApJL 390, L49 (1992)

[5] M. G. Hauser and E. Dwek, ARAA 39, 249 (2001).

[6] E. Dwek et al., ApJ 635, 784 (2005).

[7] J. P. Gardner et al., ApJL 542, L79 (2000).

[8] C. K. Xu et al., ApJL 619, L11 (2005).

[9] P. Madau and L. Pozzetti, MNRAS 312, L9 (2000).

[10] R. C. Keenan et al., ApJ 723, 40 (2010).

[11] G. G. Fazio et al., ApJS 154, 39 (2004).

[12] H. Dole et al., A\&A 451, 417 (2006).

[13] H.E.S.S. Collaboration (F. Aharonian et al.), ApJL 664, L71 (2007).

[14] P. Giommi and P. Padovani, MNRAS 268, L51 (1994).

[15] G. Ghisellini and F. Tavecchio, MNRAS 387, 1669 (2008).

[16] F. Aharonian, Very High Energy Cosmic Gamma Radiation (World Scientific Publ., Singapore, 2004).

[17] A. Reimer and M. Böttcher, Astroparticle Physics 43, 103 (2013).

[18] F. Aharonian, in proceedings of the International Cosmic Ray Conference, Vol. 27 (2001), p. I250. [arXiv:astro-ph/0112314]

[19] L. Costamante, IJMPD, 2230025C (2013) [arXiv: 1309 . 0612]

[20] A. Franceschini et al., A\&A 487, 837 (2008).

[21] R. C. Gilmore et al., MNRAS 399, 1694 (2009).

[22] J. R. Primack et al., American Institute of Physics Conference Series, Vol. 1381 (September 2011), pp. 72-83. [arXiv:1107.2566]

[23] A. Domínguez et al., MNRAS 410, 2556 (2011).

[24] F. W. Stecker et al., ApJ 648, 774 (2006).

[25] H.E.S.S. Collaboration (F. Aharonian et al.), Nature 440, 1018 (2006).

[26] H.E.S.S. Collaboration (F. Aharonian et al.), A\&A 473, L25 (2007).

[27] VERITAS Collaboration (V. A. Acciari et al.), ApJ 695, 1370 (2009). 
[28] MAGIC Collaboration (J. Albert et al.), Science 320, 1752 (2008).

[29] H.E.S.S. Collaboration (F. Aharonian et al.), A\&A 475, L9 (2007).

[30] HEGRA Collaboration (F. Aharonian et al.), A\&A 403, 523 (2003).

[31] Fermi-LAT Collaboration (M. Ackermann et al.), Science 338, 1190 (2012).

[32] G. Fossati et al., mnras 299, 433 (1998).

[33] HEGRA Collaboration (F. Aharonian et al.), A\&A 393, 89 (2002).

[34] HEGRA Collaboration (F. A. Aharonian et al.), A\&A 366, 62 (2001).

[35] D. P. Finkbeiner et al., ApJ 544, 81 (2000).

[36] R. J. Protheroe and H. Meyer, Physics Letters B 493, 1 (2000).

[37] S. Vercellone et al., in proceedings for 2012 Fermi Symposium - eConf C121028 (March 2013). [arXiv:1309.0612]

[38] CTA Consortium (B.S. Acharya et al.), Astroparticle Physics 43, 3 (2013).

[39] H.E.S.S. Collaboration (A. Abramowski et al.), A\&A 550, A4 (2013).

[40] J. Biteau and D.A. Williams, ApJ, submitted [arXiv:1502.04166]

[41] R. C. Gilmore et al., MNRAS 422, 3189 (2012).

[42] T. M. Kneiske and H. Dole, A\&A 515, A19 (2010).

[43] A. Dominguez et al., ApJ 77077 (2013)

[44] M. R. Santos et al., MNRAS 336, 1082 (2002).

[45] L. Pacciani et al., ApJ 790...45P (2014)

[46] F. A. Aharonian et al., ApJL 423, L5 (1994).

[47] A. Neronov and D. V. Semikoz, Phys. Rev. D 80, 123012 (2009).

[48] A. Neronov and I. Vovk, Science 328, 73 (2010).

[49] F. Tavecchio et al., MNRAS 406L, 70 (2010)

[50] K. Dolag et al., ApJL 727, L4 (2011).

[51] C. D. Dermer et al., ApJL 733, L21 (2011).

[52] A. M. Taylor et al., A\&A 529, A144 (2011).

[53] I. Vovk et al., ApJL 747, L14 (2012).

[54] W. Essey and A. Kusenko, Astroparticle Physics 33, 81 (2010).

[55] W. Essey et al., ApJ 731, 51 (2011).

[56] J. Einasto, International Journal of Modern Physics Conference Series 12, 100 (2012).

[57] M. Brüggen et al., ApJL 631, L21 (2005).

[58] F. Aharonian et al., Phys. Rev. D 87, 063002 (2013).

[59] A. Prosekin et al., ApJ 757, 183 (2012).

[60] HAWC Collaboration (A. Tepe et al.), Journal of Physics Conference Series 375, 052026 (2012). 